\title{
Lentivector-mediated RNAi efficiently suppresses prion protein and prolongs survival of scrapie-infected mice
}

\author{
Alexander Pfeifer, ${ }^{1,2}$ Sabina Eigenbrod, ${ }^{3}$ Saba Al-Khadra, ${ }^{1,2}$ Andreas Hofmann, ${ }^{1,2}$ \\ Gerda Mitteregger, ${ }^{3}$ Markus Moser, ${ }^{4}$ Uwe Bertsch, ${ }^{3}$ and Hans Kretzschmar ${ }^{3}$ \\ ${ }^{1}$ Institute of Pharmacology and Toxicology, University of Bonn, Bonn, Germany. ${ }^{2}$ Molecular Pharmacology, Department of Pharmacy, \\ and ${ }^{3}$ Center for Neuropathology and Prion Research, Ludwig-Maximilians-University of Munich, Munich, Germany. \\ ${ }^{4}$ Max Planck Institute of Biochemistry, Molecular Medicine, Martinsried, Germany.
}

\begin{abstract}
Prion diseases are fatal neurodegenerative diseases characterized by the accumulation of $\operatorname{PrPSc}$, the infectious and protease-resistant form of the cellular prion protein $\left(\mathrm{Pr}^{\mathrm{C}}\right)$. We generated lentivectors expressing $\operatorname{Pr}^{\mathrm{C}_{-}}$ specific short hairpin RNAs (shRNAs) that efficiently silenced expression of the prion protein gene (Prnp) in primary neuronal cells. Treatment of scrapie-infected neuronal cells with these lentivectors resulted in an efficient and stable suppression of $\mathrm{PrP}^{\mathrm{Sc}}$ accumulation. After intracranial injection, lentiviral shRNA reduced $\operatorname{Pr}^{\mathrm{C}}$ expression in transgenic mice carrying multiple copies of Prnp. To test the therapeutic potential of lentiviral shRNA, we used what we believe to be a novel approach in which the clinical situation was mimicked. We generated chimeric mice derived from lentivector-transduced embryonic stem cells. Depending on the degree of chimerism, these animals carried the lentiviral shRNAs in a certain percentage of brain cells and expressed reduced levels of $\mathrm{PrP}^{\mathrm{C}}$. Importantly, in highly chimeric mice, survival after scrapie infection was significantly extended. Taken together, these data suggest that lentivector-mediated RNA interference could be an approach for the treatment of prion disease.
\end{abstract}

\section{Introduction}

Prion diseases (transmissible spongiform encephalopathies [TSEs]) such as variant Creutzfeldt-Jakob disease (CJD) in humans, bovine spongiform encephalopathy in cattle, and scrapie in sheep are a heterogeneous group of neurodegenerative disorders. According to the protein-only hypothesis (1), the causative agent of TSEs is $\mathrm{PrP}^{\mathrm{Sc}}$, an abnormally folded, protease-resistant isoform of the cellular prion protein $\left(\mathrm{PrP}^{\mathrm{C}}\right)(2-4)$. Accumulation of $\mathrm{PrPSc}^{\mathrm{Sc}}$, which is perpetuated by an autocatalytic process (5), is the hallmark of the disease and causes neuronal dysfunction and cell death (6). So far, the majority of therapeutic approaches have targeted $\mathrm{PrPSc}$ amplification (7). In addition, several lines of evidence suggest that reducing $\mathrm{PrP}^{\mathrm{C}}$ expression could delay or even prevent disease progression $(8,9)$, because $\mathrm{PrP}^{\mathrm{C}}$-deficient $\left(P r n p^{0 / 0}\right)$ mice $(10)$ are resistant to prion disease and do not propagate infectious $\operatorname{PrP}^{\mathrm{Sc}}(8)$.

RNA interference (RNAi) is a conserved mechanism by which siRNAs specifically silence target genes (refs. in 11,12). Intracellular transcription of siRNAs can be achieved by incorporation of RNA polymerase III promoters in lentiviral vectors (refs. in 13,14$)$. Lentiviruses such as HIV are members of the family of retroviruses (15). They are able to integrate into the genome of nondividing cells, which makes them an attractive tool for gene transfer in the central nervous system (refs. in 14, 16). An important aspect of lentivectors is that they can be used for gene trans-

Nonstandard abbreviations used: CJD, Creutzfeldt-Jakob disease; dpi, days post inoculation; EGFP, enhanced GFP; LVshPrPC, lentivector carrying anti-PrPC shRNA; LVshscr, lentivector carrying control scrambled shRNA; PGK, phosphoglycerate kinase; $\mathrm{PrPC}$, cellular prion protein; $\mathrm{PrP}^{\mathrm{Sc}}$, infectious protease-resistant prion protein; RNAi, RNA interference; shRNA, short hairpin RNA; TSE, transmissible spongiform encephalopathy.

Conflict of interest: The authors have declared that no conflict of interest exists. Citation for this article: J. Clin. Invest. 116:3204-3210 (2006). doi:10.1172/JCI29236 fer in ES cells and preimplantation embryos, which is the basis of lentiviral transgenesis $(17,18)$. In this study, we combined lentiviral transgenesis with RNAi to evaluate the therapeutic potential of anti-PrPC siRNAs in mice.

\section{Results}

Lentivector-based short hairpin RNAs provide efficient and stable knock down of $P r P^{C}$ and suppress $P r P P^{S c}$ accumulation in neuronal cells. We used a lentiviral vector system derived from HIV-1 to express short hairpin RNAs (shRNAs) directed against PrPC (LVshPrPC; Figure 1A). The shRNAs are predicted to form stem-loop structures that are processed to functional siRNAs in the target cells (19). In addition, enhanced GFP (EGFP) was incorporated as a reporter gene (Figure 1A). Anti-PrPC shRNAs targeting 6 different sites in the Prnp mRNA (see Supplemental Table 1; supplemental material available online with this article; doi:10.1172/JCI29236DS1) were tested by infecting murine N2a neuroblastoma cells (Figure 1, B and C). Efficient transduction was observed, with more than $90 \%$ of cells transduced at an MOI of 10 (Figure 1B). One of the lentiviral shRNA vectors (LVsh512), which targets nt 512-532 of the Prnp coding region, decreased expression of $\mathrm{PrPC}^{\mathrm{C}}$ by more than $90 \%$ (Figure 1C). In contrast, infection of $\mathrm{N} 2 \mathrm{a}$ cells with a control lentivector carrying scrambled shRNA (LVshscr) had no significant effect $(0.2 \% \pm 8 \%$ $\operatorname{PrP}^{\mathrm{C}}$ knock down, mean $\pm \mathrm{SEM} ; n=3$; Figure $\left.1 \mathrm{C}\right)$. The $\operatorname{PrP}^{\mathrm{C}}$ knock down induced by LVsh512 was dose dependent and reached $97 \% \pm 2.7 \%(n=3)$, whereas LVshscr had no significant effect even at high doses (Figure 1D). Furthermore, lentiviral delivery of shRNA led to a stable induction of RNAi, as demonstrated by suppression of $\mathrm{PrP}^{\mathrm{C}}$ expression in LVsh512-infected cells over several passages (Supplemental Figure 1). To analyze the effect of lentiviral RNAi in primary neuronal cells, we infected cerebellar granule cells with LVsh512 (see Supplemental Figure 1). Again, Western blot analy- 
A

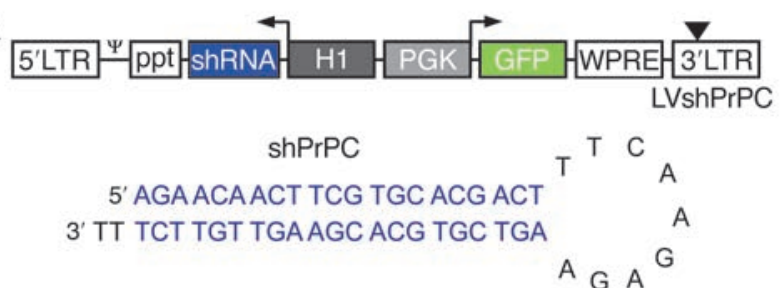

B

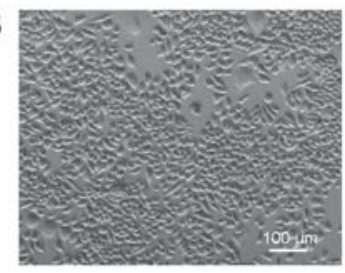

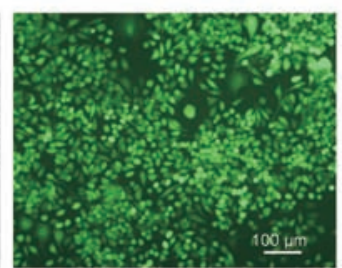
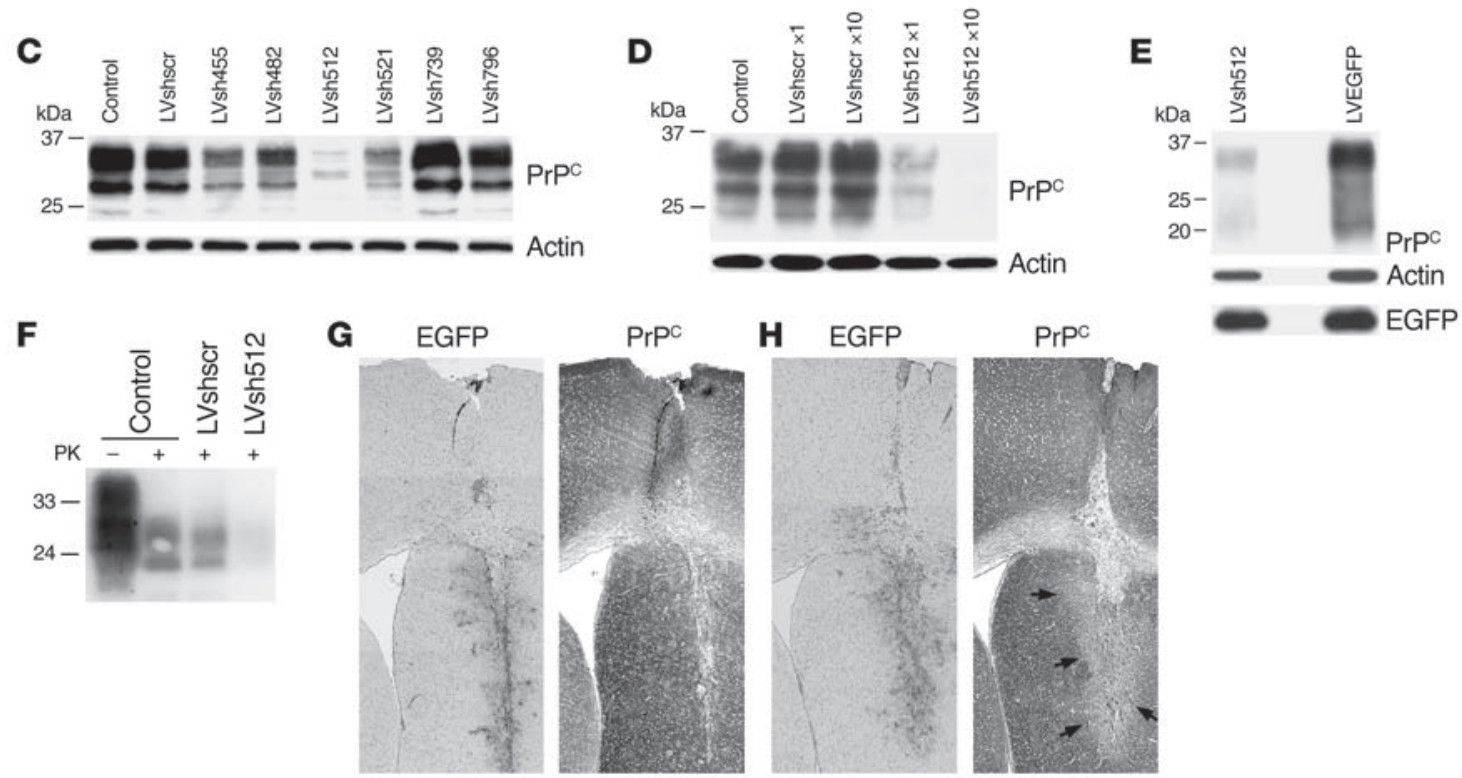

Figure 1

Targeting of $\mathrm{PrPC}^{\mathrm{C}}$ by lentivector-mediated RNAi. (A) The HIV-based lentivector (LVshPrPC, top) used carries an H1 promoter-driven shRNA cassette and a PGK-EGFP expression cassette. $\Psi$, packaging signal; ppt, polypurine tract; WPRE, woodchuck hepatitis virus posttranscriptional regulatory element; triangle, self-inactivating mutation; arrows, direction of transcription. The lentivector-encoded anti-PrPC shRNA (shPrPC, bottom) consists of 19- or 21-bp stems separated by a loop. (B) Representative bright-field (left) and fluorescence microscope (right) images of N2a cells 72 hours after transduction with LVshPrPC. (C) LVshPrPC-induced knock down of PrPC in N2a cells. Control, uninfected cells. (D) $\mathrm{PrPC}^{\mathrm{C}}$ expression in N2a cells infected with a low $(\times 1)$ and high $(\times 10)$ concentration of LVsh512 and LVshscr. $(\mathrm{E})$ Analysis of PrPC expression in granule cells 72 hours after infection with LVsh512 or a control vector carrying only the EGFP expression cassette (LVEGFP). Western blots (C-E) were probed with the indicated antibodies. (F) Effect of LVsh512 on the accumulation of protease K-resistant (PK-resistant) PrPsc in ScN2a cells. Western blot analysis 72 hours after transduction with the indicated lentivectors. (G and $\mathbf{H})$ Intracranial injection of LVshscr $(\mathbf{G})$ and LVsh512 (H) in tga20 mice overexpressing PrPC. Analysis of EGFP (left) and PrPC (right) expression 3 weeks after injection. Arrows indicate the lentivector-transduced area.

sis demonstrated an efficient reduction in $\mathrm{PrP}^{\mathrm{C}}$ expression (Figure 1E). Next, we analyzed the effect of lentiviral shRNA vectors on the accumulation of $\mathrm{PrPS}^{\mathrm{Sc}}$ in $\mathrm{N} 2 \mathrm{a}$ cells chronically infected with prions (ScN2a cells). Treatment of ScN2a cells with LVsh512 resulted in an efficient and stable reduction in $\mathrm{PrP}^{\mathrm{Sc}}$ levels as compared with those in uninfected or LVshscr-infected ScN2a cells (Figure 1F; see also Supplemental Figure 1). Thus, lentiviral shRNA vectors silenced $\mathrm{PrP}^{\mathrm{C}}$ and suppressed accumulation of $\mathrm{PrP}^{\mathrm{Sc}}$ in neuronal cells with already-established prion infection.

Reduction in $\mathrm{Pr} \mathrm{P}^{\mathrm{C}}$ expression after intracranial injection of lentiviral shRNA vectors. Next, we studied the effect of LVsh512 on $\mathrm{PrP}^{\mathrm{C}}$ expression in vivo. To analyze whether lentiviral RNAi is able to knock down even high levels of $\operatorname{PrP}^{\mathrm{C}}$, LVsh512 or LVshscr was injected stereotactically into the brain of homozygous tga20 transgenic mice. These mice carry 60 copies of the Prnp gene and express approximately 10 -fold higher levels of $\mathrm{PrPC}^{\mathrm{C}}$ than WT animals (20). Three weeks after injection, EGFP as well as $\mathrm{PrP}^{\mathrm{C}}$ expression were analyzed by immunohistochemistry (Figure 1, G and $H$ ). The lentivirus-infected area was of similar size in animals injected with
LVshscr (Figure 1G) and LVsh512 (Figure 1H), but only injection of LVsh512 induced a significant reduction in $\mathrm{PrP}^{\mathrm{C}}$ expression in the transduced area.

Knock down of $P r P^{C}$ in chimeric mice transgenic for lentiviral shRNA. To evaluate the efficacy of lentivector-mediated gene silencing in a prion disease model, we used chimeric mice. To this end, we infected 129Sv-derived ES cells with LVsh512 (Figure 2A; see also Supplemental Figure 2) and generated chimeric mice by injection of these ES cells into WT C57BL/6 blastocysts. The degree of chimerism as judged by the coat color correlated directly with the percentage of lentivirus-transduced cells as analyzed by immunohistochemistry and real-time PCR (Supplemental Figure 3). The effect of LVsh512 on $\mathrm{PrP}^{\mathrm{C}}$ expression was analyzed in 3 adult chimeric mice (nos. 1917, 1936, and 1938) derived from 1 ES cell clone (no. 512.40) that carries 2 integrants (Figure 2A). Chimera 1936 exhibited intermediate chimerism (60\% agouti coat color), while 1938 and 1917 were $80 \%$ and $90 \%$ chimeric, respectively. Direct fluorescence imaging revealed expression of the EGFP reporter throughout the chimeric brain, whereas no fluorescence was detectable in control 

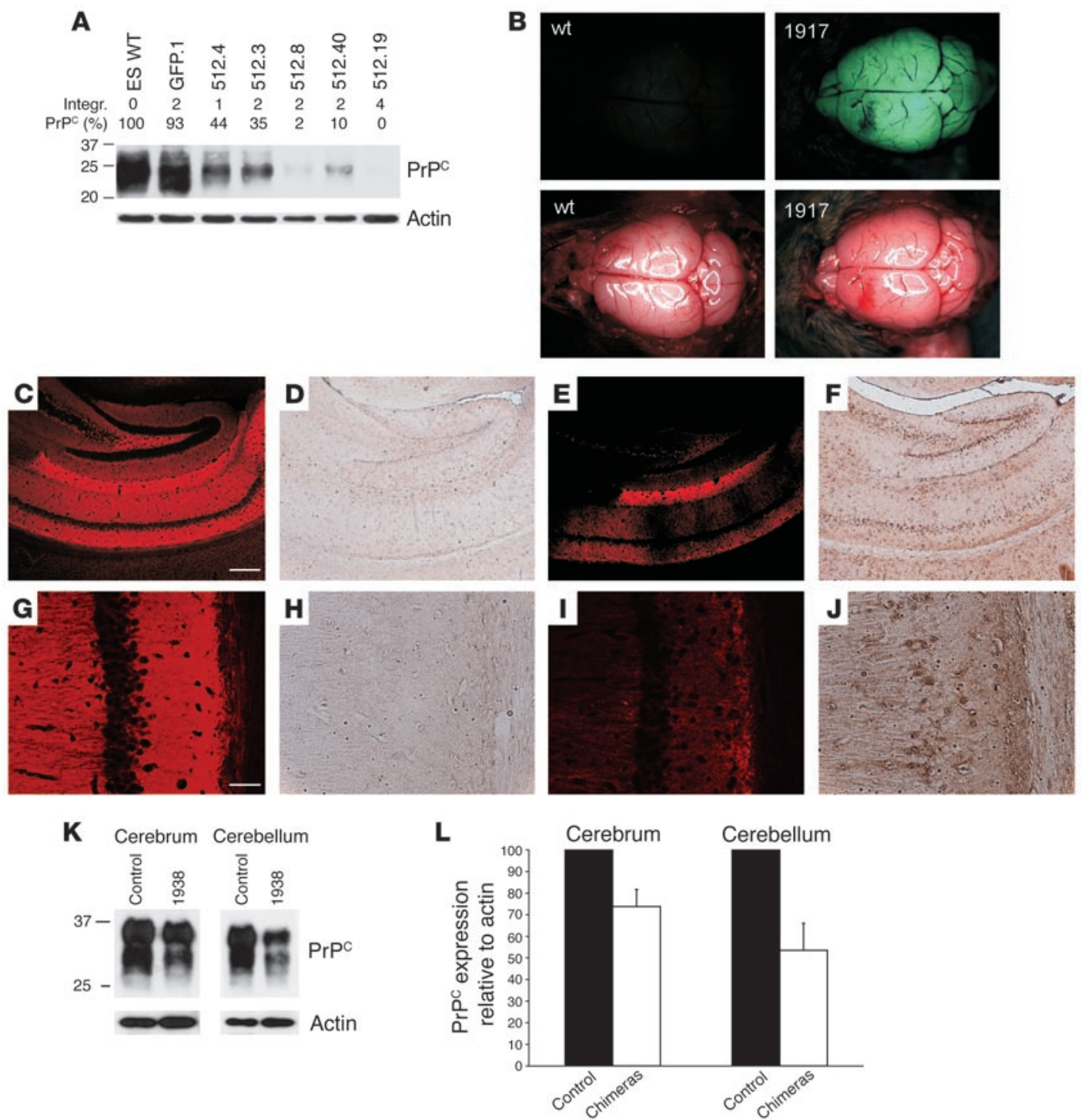

\section{Figure 2}

Silencing of PrPC in chimeric mice derived from LVsh512-infected ES cells. (A) Western blot analysis of ES cell clones infected with LVsh512 or LVEGFP. The number of integrants (Integr.) and levels of $\mathrm{PrPC}^{\mathrm{C}}$ expression ( $\mathrm{PrPC}$ ) are given above the blot. (B) Fluorescence imaging of freshly isolated brains from a control mouse (WT, left) and a transgenic animal (no. 1917, right). Shown are the fluorescence (top) and the bright-field (bottom) images. (C-J) Immunohistochemical analysis of PrPC and EGFP expression in sections of hippocampus from a transgenic (no. 1917) and an age-matched WT animal. (C and G) Analysis of PrPC expression in WT hippocampus. (E and I) Staining for PrPC revealed reduced expression of $\mathrm{PrPC}^{\mathrm{C}}$ in the chimeric hippocampus as compared with the WT. (D and $\left.\mathbf{H}\right)$ Expression of EGFP in the WT mouse. (F and $\left.\mathbf{J}\right)$ Staining for EGFP in the chimeric hippocampus, indicating the presence of the LVsh512 provirus. Higher magnifications are shown in $\mathbf{G}$ and $\mathbf{H}$ for the WT and $\mathbf{I}$ and $\mathbf{J}$ for the chimeric mouse. Scale bars: $200 \mu \mathrm{m}$ in $\mathbf{C}-\mathbf{F}$ and $50 \mu \mathrm{m}$ in $\mathbf{G}-\mathbf{J}$. (K and $\mathbf{L})$ Western blot analysis of PrPC expression in the cerebrum and cerebellum of chimeric animals.

mice (Figure 2B). Although $\mathrm{EGFP}^{+}$cells were detected in all brain regions analyzed by immunohistochemistry, the strongest staining was observed in the posterior cerebrum including the hippocampus and the cerebellum of the chimeras (Supplemental Figure 3). Immunohistochemistry of hippocampal sections revealed strong expression of $\mathrm{PrPC}^{\mathrm{C}}$ in WT mice, especially in the CA1 region (Figure 2, $\mathrm{C}$ and $\mathrm{G}$ ). In the chimeric hippocampus, a clear reduction in $\mathrm{PrP}^{\mathrm{C}}$ expression was observed (Figure 2, E and I), which correlated with the expression of EGFP (Figure 2, F and J), while no EGFPspecific staining was detected in the control (Figure 2, D and $\mathrm{H}$ ).
The coincidence of reduced $\operatorname{PrP}^{\mathrm{C}}$ expression and presence of the EGFP reporter clearly indicates that the reduction in cellular $\operatorname{PrPC}^{\mathrm{C}}$ is related to LVsh512. Furthermore, Western blot analyses of the cerebrum (containing the hippocampus) demonstrated a $26 \% \pm 8 \%$ $(n=3)$ knock down of $\mathrm{PrPC}^{\mathrm{C}}$, ranging from $15 \%$ in 1936 to $42 \%$ in the mouse with the highest degree of chimerism (no. 1917) (Figure $2, \mathrm{~K}$ and $\mathrm{L}$ ). In the cerebellum, $\operatorname{PrPC}^{\mathrm{C}}$ expression was reduced by $46 \% \pm 13 \%(n=3)$. Again, the highly chimeric mice exhibited a stronger reduction - up to $71 \%$ - than the intermediate chimeric mouse (Figure 2, $\mathrm{K}$ and $\mathrm{L}$ ), demonstrating that the ratio of 


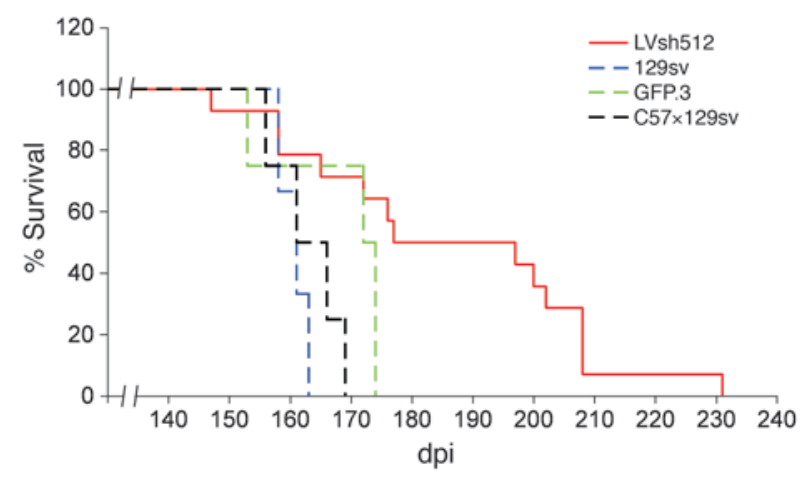

LVsh512-transgenic to nontransgenic cells (i.e., the degree of chimerism) correlated with the level of $\mathrm{PrP}^{\mathrm{C}}$ knock down in vivo.

Effect of lentivector-mediated RNAi on prion disease. To study the effects of lentiviral RNAi on prion disease, LVsh512-chimeric mice were intracerebrally inoculated with scrapie. As controls, mice chimeric for a lentivector carrying an EGFP expression cassette (GFP.3, carrying 5 integrants of LVEGFP), WT 129Sv inbred mice $(129 \mathrm{sv})$, and mice with a mixed $129 \mathrm{~Sv}-\mathrm{C} 57 \mathrm{BL} / 6$ genetic background $(\mathrm{C} 57 \times 129 \mathrm{sv})$ were inoculated in the same fashion. All control mice (GFP.3, 129sv, C $57 \times 129 \mathrm{~Sv}$ ) succumbed to prion disease within 174 days post inoculation (dpi) with scrapie (Figure 3 and Supplemental Table 2). In contrast, 64\% of the LVsh512chimeric mice lived longer than $174 \mathrm{dpi}$ (Figure 3). The mean life spans of the control groups were $167 \pm 1.2 \mathrm{dpi}(129 \mathrm{sv}), 163 \pm 2.9$ dpi (C57×129sv), and $168 \pm 5.1 \mathrm{dpi}($ GFP.3). The mean survival time of LVsh512 chimeras with low chimerism $(<35 \%$; $n=11)$ was $179 \pm 6 \mathrm{dpi}$, which was not significantly different from that of the controls (Supplemental Table 2 and Supplemental Figure 4). Two of the animals with low chimerism exhibited survival times longer than 200 days (Supplemental Table 2 and Supplemental Figure 4), indicating that expression of shRNAs in only a rather small proportion of the CNS might be sufficient to achieve protective effects if PrPC expression is reduced in structures that are essential for survival. In contrast, the highly chimeric mice (> $65 \% ; n=3$ ) survived significantly longer $(214 \pm 8$ dpi; $P<0.03)$ than the controls as well as the mice with a low degree of chimerism (Supplemental Table 2). One highly chimeric mouse ( $95 \%$ coat color chimerism) even survived scrapie infection for 231 days. Chimeric mice and controls with endstage scrapie showed similar degrees of spongiform changes and gliosis (Supplemental Table 2). However, the pathological changes in a $20 \%$ chimeric animal in end-stage scrapie were clearly more pronounced than in a $70 \%$ chimeric sibling without neurological signs that was sacrificed at the same time (Figure 4A). Western blot analysis revealed

\section{Figure 4}

\section{Figure 3}

Survival times of LVsh512-chimeric and control mice after Rocky Mountain Laboratory Inc. (RML) scrapie infection. Chimeric mice derived from LVsh512-derived ES cells survived prion disease up to 231 days. All control animals (GFP.3, green; C57×129sv, black; 129 sv, blue) died within 174 dpi with prions. Dashed lines, controls; Red line, LVsh512-chimeric mice.

a decreased amount of total PrP and reduced $\mathrm{PrP}^{\mathrm{Sc}}$ accumulation in the $70 \%$ chimeric mouse as compared with the $20 \%$ chimeric mouse (Figure 4B).

\section{Discussion}

TSEs are a large group of neurodegenerative diseases that can affect both humans and livestock. Outstanding features of TSEs are the complex pathophysiology and the fact that different routes of transmission have been identified. CJD, by far the most common human TSE, occurs either as sporadic disease of unknown etiology, as familial CJD associated with mutations in the prion gene $(P R N P)$, or as an acquired infectious disease. The latter group includes variant CJD (vCJD), which is believed to have been transmitted from cattle to humans via consumption of contaminated meat (21). In addition, recent evidence suggests that secondary transmission by blood transfusion may occur (22). It is unknown how many people are currently incubating the disease and will be affected by vCJD in the future.

Although several anti-prion compounds have been developed that are effective in the cell culture, no pharmacological treatment
A
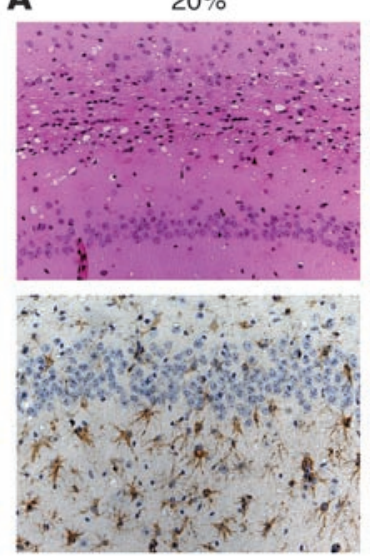

$70 \%$

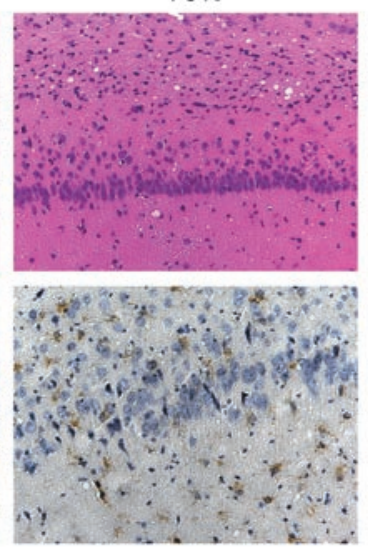

B

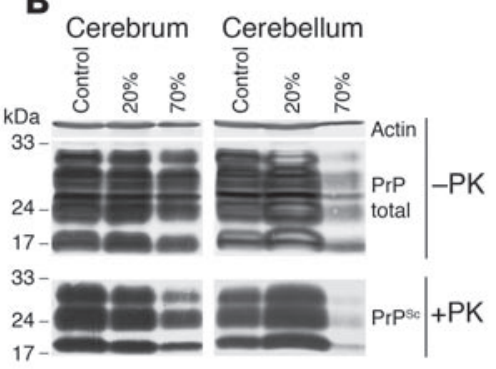

Analysis of PrPSc accumulation and neuropathological changes in prion-inoculated chimeric siblings. (A) Histological analysis of a scrapie-infected chimeric mouse (20\%) that died at $172 \mathrm{dpi}$ and a $70 \%$ chimera that was sacrificed at the same time. Analysis of spongiform changes (top) and gliosis (bottom). Coronal sections of the posterior cerebrum at the hippocampal level of the $20 \%$ (left) and $70 \%$ (right) chimeric siblings are shown. Top: H\&E staining; bottom: glial fibrillary acidic protein (GFAP) staining (brown). (B) Western blots of samples from the same chimeric mice as shown in $\mathbf{A}$ and a wildtype control. Levels of PrPsc (+PK) and total PrP (PrPtotal, -PK) in 2 different brain regions: cerebrum including hippocampus (left) and cerebellum (right). 
for TSEs exists at present $(23,24)$. Thus, there is great hope that novel therapies, including gene-based therapies, will be developed. RNAi has great potential for the therapy of a broad spectrum of diseases, including genetic disorders and infectious and neurological diseases. Among neurodegenerative disorders, prion diseases are of particular interest because of their clinical and pathogenetic features: They are the most rapidly progressive neurodegenerative disorders, they are invariably fatal, and they have a plethora of etiologies (23). Previous studies on RNAi-based therapies of neurodegenerative disorders have focused on transgenic animal models that express disease-causing toxic transgenes. Important examples are studies on polyglutamine-induced neurodegeneration $(25,26)$ and Alzheimer disease (27). An important caveat is that the siRNAs used in these mouse models of neurodegenerative diseases selectively targeted the disease-causing foreign transgenes, thus avoiding the knock down of the corresponding normal murine gene. This is quite different from a realistic therapeutic setting in humans, where the shRNA used could reduce expression of both the diseased and the normal allele, which could lead to unwanted side effects. Similarly, the vast majority of studies on the use of RNAi for the treatment of infectious diseases have targeted the infectious agent, e.g., viral genes involved in replication (28-30). Given the recently described virus-encoded counter-defense mechanisms (refs. in 31), using siRNAs against essential host genes might be an attractive alternative or additional therapeutic strategy to targeting the infectious agent.

In our study, we targeted the host factor essential for replication of the infectious agent, i.e., the gene coding for the PrPC . Direct transfection of siRNAs achieved only a transient reduction in $\mathrm{Pr} \mathrm{PC}^{\mathrm{C}}$ expression in cell culture models $(32,33)$. On the other hand, vectors derived from lentiviruses and adeno-associated virus have been successfully used for long-term expression of siRNAs in vivo $(25-27,34,35)$. Lentiviruses efficiently integrate their genome into neuronal cells, which makes them an attractive tool for the development of therapies for neurological disorders $(13,14,36)$. The lentiviral shRNA vector that resulted in the most effective silencing of $\mathrm{PrPC}^{\mathrm{C}}$ in neuroblastoma cells (LVsh512) also efficiently reduced $\mathrm{Pr}^{\mathrm{C}}$ expression in primary neuronal cells. Importantly, LVsh512 suppressed accumulation of $\mathrm{PrPsc}^{\mathrm{S}}$ in neuronal cells with already established, chronic scrapie infection. After intracranial injection of LVsh512, we observed reduced $\mathrm{PrP}^{\mathrm{C}}$ expression in the vicinity of the injection site, demonstrating the potential of lentivectors to silence $\mathrm{PrP}^{\mathrm{C}}$ in vivo. Injection of viral siRNA vectors into a defined region of the brain (i.e., the striatum or the hippocampus) has been shown to improve behavioral phenotypes in murine models of other neurodegenerative diseases (25, 27). However, TSEs are characterized by disseminated degeneration of neuronal cells throughout the brain. Therefore, we combined the use of lentiviral shRNA vectors and ES cell technology, establishing what we believe to be a novel way to test lentiviral shRNA therapies in animals. Through transfer of ES cells that carry the lentiviral shRNA vectors into WT blastocysts, chimeric mice can be generated that carry shRNA-expressing cells as well as WT cells. In contrast, transgenic animals that are generated through germ-line transmission carry the transgene in all their cells. However, somatic gene transfer in all cells of the brain is not feasible in a therapeutic setting, and germ-line gene transfer can not be done in humans. Thus, chimeric mice are intriguing test models for RNAi-based gene therapy. Scrapie-infected mice chimeric for lentivirally transduced cells exhibited a significantly prolonged survival, which correlated with the number of cells carrying the lentiviral shRNA vector.
Our data not only provide a rationale for RNAi-based prion therapies, but also underline the importance of efficient delivery of lentiviral shRNA vectors for the successful use of this technology in the human CNS. Through stereotactical procedures, only a limited number of target CNS regions can be injected with lentivectors. Catheter placement and/or pharmacological opening of the blood-brain barrier to lentiviral vectors might be ways for a more widespread transduction of the CNS. In order for therapy with lentiviral shRNA vectors to be successful, it will also be necessary to design shRNA that achieves efficient and selective knock down of the target gene and to develop techniques to efficiently transduce the target cells, which requires high-titer vector preparations.

The biomedical relevance of antiprion lentiviral shRNAs is not restricted to a therapeutic use in humans. Lentivirus-mediated RNAi is also of interest for targeting of Prnp in livestock. Lentiviral transgenesis in livestock (for review, see refs. 37-39) is based either on direct transduction of preimplantation embryos (40-42) or on creation of lentivirus-transgenic cells for somatic cell nuclear transfer $(42,43)$. Silencing of $\operatorname{PrP}^{\mathrm{C}}$ expression in sheep and cattle by lentiviral anti-PrPC $\mathrm{PhRNAs}^{\mathrm{C}}$ (42) is a promising alternative to disruption of the PRNP gene by gene targeting (44). Prion-resistant livestock could reduce the risk of TSE transmission to humans and would be especially interesting for the production of biomedical products (gene pharming).

Taken together, our results indicate that the use of chimeric mice derived from lentivirus-transduced ES cells is a novel approach that allows the evaluation of critical parameters, such as efficacy and vector safety issues, of lentivector-based gene therapy strategies in the whole animal. Lentiviral shRNA vectors are powerful tools to address different aspects of TSEs, including basic research, applied sciences, as well as the development of therapeutic strategies.

\section{Methods}

Lentivector design and production. Target sites for RNAi were selected using online programs (oligoengine, http://www.oligoengine.com/, and Dharmacon, http://www.dharmacon.com/sigenome/default.aspx\#cpnlPanel2). Oligonucleotides (MWG Biotech) were cloned into the H1-driven expression cassette. The lentivector backbone (LVEGFP), which carries EGFP under the control of the human phosphoglycerate kinase (PGK) promoter, was described previously (45). The H1-shRNA expression cassette was inserted between the polypurine tract and the PGK promoter in opposite transcriptional direction of the PGK-EGFP cassette. For intracranial injections, the PGK promoter of the EGFP expression cassette was replaced by a CMV promoter. Recombinant lentivirus was produced as recently described (18).

Cell culture, in vitro infection, and generation of chimeric mice. The N2a neuroblastoma cell line was cultured in DMEM (Invitrogen), supplemented with $10 \%$ heat inactivated FCS (Biochrom AG) and antibiotics $(100 \mathrm{U} / \mathrm{ml}$ penicillin $\mathrm{G}$ and $100 \mathrm{mg} / \mathrm{ml}$ streptomycin; Biochrom AG). Preparation of cerebellar granule cells was performed as previously described (46). Briefly, the cerebella were dissociated in HBSS medium (PAN Biotech GmbH) containing 1\% trypsin (Biochrom AG), washed with Dulbecco's PBS (PAN Biotech $\mathrm{GmbH}$ ) and plated on 6-well plates coated with poly-D-lysine (50 $\mathrm{mg} / \mathrm{ml}$; Sigma-Aldrich) in Dulbecco's Minimal Essential Medium (PAA) supplemented with $10 \% \mathrm{FCS}, 2 \mathrm{mM}$ glutamine, $20 \mathrm{mM} \mathrm{KCl}$, and antibiotics. To test different shRNAs (LVshPrPCs), cells were infected overnight (at 40\%-50\% confluence) with equal amounts of virus particles (from each virus preparation) and harvested 72 hours later.

Murine R1 ES cells (47) were cultured on feeder cells (murine embryonic fibroblasts) in DMEM, supplemented with $20 \%$ FCS, $7 \mu \mathrm{l} / 1 \beta$-mer- 
captoethanol, and 1,000 U/ml LIF (ESGRO; Chemicon International). Chimeric mice were generated as previously described (18). In brief, stable transgenic ES cell clones were injected into C57BL/ 6 blastocysts to generate black-agouti chimeras.

Western blotting. Cells were lysed in protease-inhibiting solution $(0.5 \%$ octylphenyl-polyethylene glycol, $150 \mathrm{mM} \mathrm{NaCl}, 0.5 \%$ sodium deoxycholate, $50 \mathrm{mM}$ Tris, $\mathrm{pH} 7.4$ and supplemented with complete protease inhibitors [Roche Diagnostics]) and directly used for Western blotting.

Tissues were homogenized in lysis buffer $(0.5 \% \mathrm{NP}-40,100 \mathrm{mM} \mathrm{NaCl}$, $10 \mathrm{mM}$ EDTA, $0.5 \%$ sodium deoxycholate, $10 \mathrm{mM}$ Tris, $\mathrm{pH} 7.6$ and supplemented with complete protease inhibitors [Roche Diagnostics]). After separation by $15 \%$ SDS-PAGE, proteins were transferred to a polyvinylidene difluoride membrane (Immobilon-P Transfer Membrane; Millipore). For $\operatorname{PrP}^{\mathrm{C}}$ detection, membranes were incubated at $4{ }^{\circ} \mathrm{C}$ overnight with anti-PrP ${ }^{\mathrm{C}}$ antibodies (6H4 monoclonal; Prionics). EGFP was detected using monoclonal antibodies against EGFP (Living Colors; BD Biosciences - Clontech). $\operatorname{PrPC}^{\mathrm{C}}$ and EGFP signals were visualized using secondary peroxidase-conjugated antibodies (Dianova) and an enhanced chemiluminescence detection kit (Amersham Pharmacia). To measure protein loading, antibodies directed against $\beta$-actin (Sigma-Aldrich) were used.

Detergent solubility assay. For detergent solubility assay, cells or tissues were lysed ( $0.5 \%$ Triton X-100 and $0.5 \%$ sodium deoxycholate in PBS). Cells were incubated for 1 hour at $4{ }^{\circ} \mathrm{C}$ with $1 \mu \mathrm{g}$ proteinase $\mathrm{K}$ (Roche Diagnostics) per $300 \mu \mathrm{g}$ protein. The proteinase $\mathrm{K}$ digestion was terminated by boiling in Laemmli sample buffer (5 mM EDTA, pH 8.0, 1.5\% SDS, 10\% glycine, $0.025 \%$ bromphenol blue, $1.5 \% \beta$-mercaptoethanol, $0.8 \mathrm{M}$ urea).

Intracranial injections. For intracranial injections, male tga20 mice (20) were placed in a stereotactic frame (SR-N5 with MA6 auxiliary ear bars; Narishige) under anesthesia $(0.15 \mathrm{ml} / 30 \mathrm{~g}$ body weight i.p. of $20 \mathrm{mg} / \mathrm{ml}$ xylazine, $115.34 \mathrm{mg} / \mathrm{ml}$ ketamine hydrochloride, $13.56 \mathrm{mg} / \mathrm{ml}$ acepromazine maleate in $0.9 \% \mathrm{NaCl}$ ). The striatum was targeted unilaterally on the right hemisphere using a $30-\mathrm{G}$ needle (Hamilton) connected to a Hamilton $5-\mu l$ syringe. The stereotactic coordinates for the microinjections were: +0.4 $\mathrm{mm}$ anterior, $+2.0 \mathrm{~mm}$ lateral, and $-3.5 \mathrm{~mm}$ caudal to bregma. Lentiviral vectors $\left(\sim 5 \times 10^{8}\right.$ transducing particles $\left./ \mathrm{ml}\right)$ were injected at a rate of $1 \mu \mathrm{l} / \mathrm{min}$ over 2 minutes. Mice were sacrificed after 3 weeks.

Scrapie inoculation of mice. LVsh512-chimeric mice (10\%-95\% chimerism) and control mice $(129 \mathrm{sv}, \mathrm{C} 57 \times 129 \mathrm{sv}$, and GFP.3) were inoculated intracerebrally with $30 \mu \mathrm{l}$ of a $10 \%$ (wt/vol) clarified suspension of brain in MEM (Invitrogen) from mice terminally affected with Rocky Mountain Laboratory Inc. (RML) scrapie prions. Mice were monitored for clinical signs of scrapie and sacrificed when the terminal state of disease was reached.

Genomic DNA extraction and Southern blot hybridization. Cells were treated with extraction buffer (100 mM Tris, $5 \mathrm{mM}$ EDTA, $0.2 \%$ SDS, $200 \mathrm{mM} \mathrm{NaCl}$, and $0.1 \mathrm{mg} / \mathrm{ml}$ proteinase $\mathrm{K}$ ) at $55^{\circ} \mathrm{C}$ overnight. Genomic DNA was precipitated with isopropanol, dried, and dissolved in an appropriate volume of $\mathrm{H}_{2} \mathrm{O}$. For Southern blotting, the DNA was digested with BamHI and separated by gel electrophoresis. The DNA was then transferred to Gene Screen Plus Hybridization Transfer Membranes (PerkinElmer) and hybridized with a full-length ${ }^{32} \mathrm{P}-$ labeled EGFP cDNA probe. The signals were visualized in the BAS 1000 Radioluminography Laser Scanner (raytest).

FACS analysis. LVshPrPC-infected N2a cells were fixed in 4\% paraformaldehyde (PFA), and fluorescence was measured by FACScan (BD).

Fluorescence imaging. Green fluorescence was observed in unfixed brains using a Schott KL 2500 light source and a 485 -nm filter (Zeiss). The fluorescence was visualized using a long-pass filter (HQ500; Zeiss), and images were taken with an AxioCam HRc (Zeiss).

Immunohistochemistry. GFP stainings of brain sections derived from intracranial injections were performed using monoclonal anti-GFP antibodies (Santa Cruz Biotechnology Inc.). For PrPC stainings, monoclonal CDC1 antibodies (raised against recombinant full-length mouse PrP) were used on a Benchmark staining machine with a standard alkaline phosphatase anti-alkaline phosphatase detection system according to the manufacturer's instructions (Ventana Medical Systems Inc.).

Paraffin sections of hippocampus from chimeric mice were incubated with polyclonal antibodies against EGFP (Abcam), followed by incubation with secondary biotinylated antibodies (anti-goat; Dianova), and stained with $\mathrm{ABC}$ solution (Vector Laboratories) and 3',3-diaminobenzidine (DAB; $270 \mu \mathrm{g} / \mathrm{ml}$; Sigma-Aldrich). $\operatorname{PrPC}^{\mathrm{C}}$ stainings of consecutive sections were performed as described for brain sections derived from intracranial injections. Brain tissues of scrapie-infected mice were fixed in 4\% PFA, paraffin embedded, and decontaminated in formic acid. Gliosis and spongiosis were visualized using anti-glial fibrillary acidic protein polyclonal antiserum (Dako) and H\&E staining, respectively.

For immunofluorescence analysis of neuronal cells, brain sections were incubated with NF200 (Sigma-Aldrich) antibodies followed by incubation with TRITC-conjugated secondary antibodies. Subsequently, sections were incubated with anti-EGFP primary antibodies (Santa Cruz Biotechnology Inc.) and FITC-conjugated secondary antibodies. Finally, cell nuclei were stained with DAPI. Numbers of cells (total, NF200-positive, and EGFP-positive) were determined using cell ${ }^{\mathrm{D}}$ software (Olympus) on 4 randomly chosen sections.

Quantification of transgenic cells by real-time PCR analysis. The real-time PCR analysis was performed with the iQ5 Real-Time PCR Detection System (Bio-Rad) and the IQ Multiplex Powermix (Bio-Rad) according to the manufacturer's instructions. The amplification conditions were 2 minutes at $50^{\circ} \mathrm{C}, 10$ seconds at $95^{\circ} \mathrm{C}$, a 2 -step cycle of $95^{\circ} \mathrm{C}$ for 15 seconds, and $60^{\circ} \mathrm{C}$ for 60 seconds for a total of 40 cycles. To assay the number of transgenic cells in the brain of chimeric mice, we isolated genomic DNA (see above). We used primers that amplify the region of the integrated lentiviral DNA between the left LTR sequence and the $5^{\prime}$ end of the gag gene (late RT as described in ref. 48). The late RT probe (48) was labeled with 6-FAM at the $5^{\prime}$ end and Eclipse Dark Quencher (EDQ) at the $3^{\prime}$ end (Eurogentec). To measure DNA loading, we used primers (ForBlr, 5'-CGGAGCTCAACCGAGACCT-3' and RevBlr, 5'-TGCAAAAGGCAGGATGAAGA-3') and a probe (5'-TexasRed-CTGTTCCACCTCGCAGTAGCCGAC-EDQ-3'; Eurogentec) for the gene of Burkitt lymphoma receptor 1 (BLR1). We performed a duplex PCR with both PCR reactions occurring in the tube. The final primer and probe concentrations, in a total volume of $25 \mu 1$, were $300 \mathrm{nM}$ and $200 \mathrm{nM}$, respectively. DNA samples were measured in triplicate, and the means of the threshold cycle values were taken to quantify the number of transgenic cells relative to the total number of cells using the BLR1 gene as a control for DNA loading.

All procedures were in accordance with the recommendation of the Tierschutz-Informations-Zentrum für die Biomedizinische Forschung der Medizinischen Fakultät (Animal Welfare Information Centre for Biomedical Research of the Faculty of Medicine) of Ludwig-Maximilians-Universität in Munich. All experiments were approved by the Regierung von Oberbayern (Munich, Germany).

Statistics. For statistical analysis, data were analyzed using the unpaired 2-tailed Student's $t$ test. $P \leq 0.05$ was considered to be statistically significant.

\section{Acknowledgments}

This work was supported by grants from the Deutsche Forschungsgemeinschaft (PF 301/8-2). We thank Luigi Naldini (San Raffaele Telethon Institute for Gene Therapy [HSR-TIGET], San Raffaele Scientific Institute, Milan) for providing lentiviral plasmids. We thank Eva Grasbon-Frodl for help with stereotaxy, Antje Strack for help with granule cell preparation, Claudia Pace for performing scrapie infections, Rüdiger Schmalzbauer for generation of 
the CDC1 antibody, Christina Priller for help with fluorescence microscopy, as well as Heidi Sebald, Andrea Kabermann, Janina Mielke, and Michael Schmidt for expert technical assistance.

Received for publication May 30, 2006, and accepted in revised form August 29, 2006.

Address correspondence to: Alexander Pfeifer, Institute of Pharmacology and Toxicology, University of Bonn, Reuterstr. 2B, 53113
Bonn, Germany. Phone: 49-228-735410; Fax: 49-228-735404; E-mail: alexander.pfeifer@uni-bonn.de. Or to: Hans Kretzschmar, Center for Neuropathology and Prion Research, Ludwig Maximilians University of Munich, Feodor-Lynen-Strasse 23, 81377 Munich, Germany. Phone: 49-89-2180-78001; Fax: 49-89-218078037; E-mail: hans.kretzschmar@med.uni-muenchen.de.

Alexander Pfeifer, Sabina Eigenbrod, and Saba Al-Khadra contributed equally to this work.
1. Prusiner, S.B. 1982. Novel proteinaceous infectious particles cause scrapie. Science. 216:136-144.

2. Griffith, J.S. 1967. Self-replication and scrapie. Nature. 215:1043-1044.

3. Bolton, D.C., McKinley, M.P., and Prusiner, S.B. 1982. Identification of a protein that purifies with the scrapie prion. Science. 218:1309-1311.

4. McKinley, M.P., Bolton, D.C., and Prusiner, S.B. 1983. A protease-resistant protein is a structural component of the scrapie prion. Cell. 35:57-62.

5. Bieschke, J., et al. 2004. Autocatalytic self-propagation of misfolded prion protein. Proc. Natl. Acad. Sci. U. S. A. 101:12207-12211.

6. Giese, A., and Kretzschmar, H.A. 2001. Prioninduced neuronal damage - the mechanisms of neuronal destruction in the subacute spongiform encephalopathies. Curr. Top. Microbiol. Immunol. 253:203-217.

7. Aguzzi, A., Glatzel, M., Montrasio, F., Prinz, M., and Heppner, F.L. 2001. Interventional strategies against prion diseases. Nat. Rev. Neurosci. 2:745-749.

8. Bueler, H., et al. 1993. Mice devoid of PrP are resistant to scrapie. Cell. 73:1339-1347.

9. Mallucci, G., et al. 2003. Depleting neuronal PrP in prion infection prevents disease and reverses spongiosis. Science. 302:871-874.

10. Bueler, H., et al. 1992. Normal development and behaviour of mice lacking the neuronal cell-surface PrP protein. Nature. 356:577-582.

11. Hannon, G.J., and Rossi, J.J. 2004. Unlocking the potential of the human genome with RNA interference. Nature. 431:371-378.

12. Tuschl, T. 2002. Expanding small RNA interference. Nat. Biotechnol. 20:446-448.

13. Singer, O., Yanai, A., and Verma, I.M. 2004 Silence of the genes. Proc. Natl. Acad. Sci. U. S. A. 101:5313-5314.

14. Wiznerowicz, M., and Trono, D. 2005. Harnessing HIV for therapy, basic research and biotechnology. Trends Biotechnol. 23:42-47.

15. Goff, S.P. 2001. Retroviridae: the retroviruses and their replication. In Fields virology. P.M. Howley, et al., editors. Lippincott-Raven Publishers. Philadelphia, Pennsylvania, USA. 1871-1939.

16. Pfeifer, A., and Verma, I.M. 2001. Gene therapy: promises and problems. Annu. Rev. Genomics Hum. Genet. 2:177-211.

17. Lois, C., Hong, E.J., Pease, S., Brown, E.J., and Baltimore, D. 2002. Germline transmission and tissue-specific expression of transgenes delivered by lentiviral vectors. Science. 295:868-872.

18. Pfeifer, A., Ikawa, M., Dayn, Y., and Verma, I.M.
2002. Transgenesis by lentiviral vectors: lack of gene silencing in mammalian embryonic stem cells and preimplantation embryos. Proc. Natl. Acad. Sci. U. S. A. 99:2140-2145

19. Brummelkamp, T.R., Bernards, R., and Agami, R. 2002. A system for stable expression of short interfering RNAs in mammalian cells. Science. 296:550-553.

20. Fischer, M., et al. 1996. Prion protein (PrP) with amino-proximal deletions restoring susceptibility of PrP knockout mice to scrapie. EMBO J. 15:1255-1264.

21. Ironside, J.W. 2003. Variant Creutzfeldt-Jakob disease. Vet. Res. Commun. 27(Suppl. 1):11-13.

22. Llewelyn, C.A., et al. 2004. Possible transmission of variant Creutzfeldt-Jakob disease by blood transfusion. Lancet. 363:417-421.

23. Aguzzi, A., Heikenwalder, M., and Miele, G. 2004. Progress and problems in the biology, diagnostics, and therapeutics of prion diseases. J. Clin. Invest. 114:153-160. doi:10.1172/JCI200422438.

24. Trevitt, C.R., and Collinge, J. 2006. A systematic review of prion therapeutics in experimental models. Brain. 129:2241-2265.

25. Harper, S.Q., et al. 2005. RNA interference improves motor and neuropathological abnormalities in a Huntington's disease mouse model. Proc. Natl. Acad. Sci. U. S. A. 102:5820-5825.

26. Xia, H., et al. 2004. RNAi suppresses polyglutamine-induced neurodegeneration in a model of spinocerebellar ataxia. Nat. Med. 10:816-820.

27. Singer, O., et al. 2005. Targeting BACE1 with siRNAs ameliorates Alzheimer disease neuropathology in a transgenic model. Nat. Neurosci. 8:1343-1349.

28. Lee, N.S., et al. 2002. Expression of small interfering RNAs targeted against HIV-1 rev transcripts in human cells. Nat. Biotechnol. 20:500-505.

29. Kumar, P., Lee, S.K., Shankar, P., and Manjunath, N. 2006. A single siRNA suppresses fatal encephalitis induced by two different flaviviruses. PLoS Med. 3:e96.

30. Bai, F., et al. 2005. Use of RNA interference to prevent lethal murine west nile virus infection. J. Infect. Dis. 191:1148-1154.

31. Manjunath, N., Kumar, P., Lee, S.K., and Shankar, P. 2006. Interfering antiviral immunity: application, subversion, hope? Trends Immunol. 27:328-335.

32. Daude, N., Marella, M., and Chabry, J. 2003. Specific inhibition of pathological prion protein accumulation by small interfering RNAs. J. Cell Sci. 116:2775-2779.

33. Tilly, G., Chapuis, J., Vilette, D., Laude, H., and
Vilotte, J.L. 2003. Efficient and specific downregulation of prion protein expression by RNAi. Biochem. Biophys. Res. Commun. 305:548-551.

34. Ralph, G.S., et al. 2005. Silencing mutant SOD1 using RNAi protects against neurodegeneration and extends survival in an ALS model. Nat. Med. 11:429-433.

35. Raoul, C., et al. 2005. Lentiviral-mediated silencing of SOD1 through RNA interference retards disease onset and progression in a mouse model of ALS. Nat. Med. 11:423-428.

36. Bonini, N.M., and La Spada, A.R. 2005. Silencing polyglutamine degeneration with RNAi. Neuron. 48:715-718.

37. Pfeifer, A. 2004. Lentiviral transgenesis. Transgenic Res. 13:513-522.

38. Whitelaw, C.B. 2004. Transgenic livestock made easy. Trends Biotechnol. 22:157-159; discussion 159-160.

39. Fassler, R. 2004. Lentiviral transgene vectors. $E M B O$ Rep. 5:28-29.

40. Hofmann, A., et al. 2003. Efficient transgenesis in farm animals by lentiviral vectors. EMBO Rep. 4:1054-1060.

41. Whitelaw, C.B., et al. 2004. Efficient generation of transgenic pigs using equine infectious anaemia virus (EIAV) derived vector. FEBS Lett. 571:233-236.

42. Golding, M.C., Long, C.R., Carmell, M.A., Hannon, G.J., and Westhusin, M.E. 2006. Suppression of prion protein in livestock by RNA interference. Proc. Natl. Acad. Sci. U. S. A. 103:5285-5290.

43. Hofmann, A., et al. 2004. Generation of transgenic cattle by lentiviral gene transfer into oocytes. Biol. Reprod. 71:405-409.

44. Denning, C., et al. 2001. Deletion of the alpha$(1,3)$ galactosyl transferase (GGTA1) gene and the prion protein $(\mathrm{PrP})$ gene in sheep. Nat. Biotechnol. 19:559-562.

45. Follenzi, A., Ailles, L.E., Bakovic, S., Geuna, M., and Naldini, L. 2000. Gene transfer by lentiviral vectors is limited by nuclear translocation and rescued by HIV-1 pol sequences. Nat. Genet. 25:217-222.

46. Brown, D.R., Schmidt, B., and Kretzschmar, H.A. 1996. Role of microglia and host prion protein in neurotoxicity of a prion protein fragment. Nature. 380:345-347.

47. Nagy, A., Rossant, J., Nagy, R., Abramow-Newerly, W., and Roder, J.C. 1993. Derivation of completely cell culture-derived mice from early-passage embryonic stem cells. Proc. Natl. Acad. Sci. U. S. A. 90:8424-8428.

48. Butler, S.L., Hansen, M.S., and Bushman, F.D. 2001. A quantitative assay for HIV DNA integration in vivo. Nat. Med. 7:631-634. 